\title{
Síndrome de Rapunzel: tricobezoar gastroduodenal
}

\author{
DAVID LOJA ${ }^{1,3}$, JOSÉ ALVIZURII,3, MARICELA VILCA 4 , MARIO SÁNCHEZ 2,3 \\ ${ }^{1} D$ epartamento de M edicina Interna y ${ }^{2} D$ epartamento de Cirugía General del \\ ${ }^{3} \mathrm{H}$ ospital Arzobispo Loayza, Lima - Perú. ${ }^{4}$ C entro M édico Quirúrgico Clínica Chosica, EsSalud.
}

\begin{abstract}
RE SUMEN
EI Síndrome de Rapunzel es una forma inusual de tricobezoar gástrico con extensión al intestino. Está compuesto de pelo, moco y fragmentos de alimentos en descomposición. Reportamos el caso de una adolescente de 18 años de edad que es admitida en nuestra institución con antecedente de depresión mayor, en tratamiento irregular en el año previo e historia de 5 meses de náuseas, vómitos y pérdida ponderal de $15 \mathrm{~kg}$. Los hallazgos del examen incluyeron distensión y dolor abdominal. Tenía anemia severa y en la serie gastroduodenal mostró defecto de relleno gástrico. EI diagnóstico fue confirmado con la gastroscopia. La gastrotomía reveló un gran tricobezoar con extensión duodenal, el que fue extraído. Fue dada de alta sin complicaciones.
\end{abstract}

Palabras clave: Bezoares; tricotilomanía; enfermedades gastrointestinales.

\section{RAPUNZEL SY NDROME: GASTRODUODENAL TRICHOBEZOAR SUM MARY}

Rapunzel syndrome is a rare form of gastric trichobezoar extending to the bowel. It is composed of hair, mucus and decaying food particles. We report the case of an 18 year-old girl who was admitted to our institution with past history of irregular treatment of major depression during the previous year and history of five months of nauseas, vomiting and $15 \mathrm{~kg}$ weight loss. On examination findings included abdominal tumor mass, distention and pain. She presented severe anemia and the gastroduodenal $x$-rays showed gastric filling defects. Diagnosis was confirmed by gastroscopy and gastrotomy revealed a large tricobezoar with duodenal projection, that was removed. She did not present complications on discharge.

Key words: Bezoars; trichotillomania; gastrointestinal diseases.

\section{INTRODUCCIÓN}

Se denomina bezoares a los cuerpos extraños que se pueden formar en el estómago por la ingestión de sustancias no digeribles, tales como pelos, fibras de coco, residuo de dátiles, fibras de nísperos no maduros, papeles, almidón, resinas, lacas, alquitrán y otros ${ }^{(1-5)}$, Tabla 1.

Correspondencia:

Dr. David Loja Oropeza

Reynolds 114 - 101

Lima 41, Perú

E-mail: davidloja@hotmail.com
U na forma poco común, pero eventualmente fatal de tricobezoar, es el síndrome de Rapunzel (SR), denominación que evoca el cuento de los hermanos W ilhelm y J acob G rimm que, en 1812, escribieron la historia sobre Rapunzel; una joven que lanzaba su larga cabellera para que su enamorado -el príncipe- subiera a la torre donde se encontraba prisionera ${ }^{(6-18)}$. Este síndrome se caracteriza porque el cuerpo del tricobezoar se localiza en el estómago y su cola en el intestino delgado y/o en el colon derecho, produce obstrucción intestinal al ta o baja y ocurre en pacientes psiquiátricos que acusan tricofagia $\left({ }^{19}\right)$. Reportamos un caso y revisamos la literatura. 
Tabla 1. Clasificación de los bezoares.
1.-Tricobezoar: Pelo
2.-Fitobezoar
a.-Iniciobezoar: Fibras de coco
b.-Bezoar oriental: Residuo de dátiles
c.-Diospirobezoar: Fibras de nísperos verdes
d.-Bezoar por papel

3.-Quimiobezoar

a.-Halibezoar: Residuo de drogas

b.-Amilobezoar: Almidón

c.-Resinobezoar: Resinas

d.-Halobezoar: Sustancias orgánicas

e.-Hemobezoar: Sangre

f.-Lactobezoar: Leche

g.-Quimiobezoar por lacas

h.-Quimiobezoar por alquitrán

i.-Mixtos

\section{CASO CLÍNICO}

Paciente mujer de 18 años de edad, natural y procedente de Lima, soltera, que ingresó por Emergencia en julio de 1997, con historia de aproximadamente 4 años de náuseas, vómitos alimenticios posprandiales inmediatos, plenitud gástrica y pérdida ponderal de $15 \mathrm{~kg}$ en los últimos 5 meses.

A ntecedente de depresión mayor en tratamiento con amitriptilina, en forma irregular, durante el año previo. Procedía de un entorno familiar con múltiples conflictos y estado socioeconómico pobre. Negó tricotilomanía y tricofagia.

A I examen físico, las constantes vitales fueron normales. Había marcado adelgazamiento y palidez, edema maleolar leve y ausencia de adenomegalias. M urmullo vesicular pasaba bien en ambos campos pulmonares, sin ruidos agregados. Los ruidos cardíacos eran rítmicos, de buena intensidad, soplo sistólico de grado II/V I multifocal. El abdomen estaba distendido, blando, depresible. Se palpaba una tumoración de aproximadamente $10 \times 15 \mathrm{~cm}$ localizada en epigastrio y mesogastrio, de consistencia algo incrementada, dolorosa a la palpación superficial y profunda. No había visceromegalia y los ruidos hidroaéreos estaban presentes. EI resto del examen no fue contributorio.

Exámenes auxiliares: Hemoglobina $5,7 \mathrm{mg} /$ $\mathrm{dL}$, leucocitos $6800 \mathrm{~mL}$ (abastonados 2, segmentados 63 , eosinófilos 10 , basófilos 2 , monocitos 0 , linfocitos 23), plaquetas 155000 / $\mathrm{mL}$, velocidad de sedimentación globular 22 $\mathrm{mm} / \mathrm{h}$, deshidrogenasa láctica $110 \mathrm{U} / \mathrm{L}$, creatinina $0,56 \mathrm{mg} / \mathrm{dL}$. El estudio coproparasitológico seriado fue negativo y las pruebas de función hepática normales. En la ecografía abdominal se apreció una masa tumoral que impresionó ser dependiente del hígado. La radiografía de estómago y duodeno mostró gran tumoración en el lumen gástrico, con adsorción de bario en su superficie, sugerente de bezoar; no se descartó proceso neoformativo (Figura 1).

L a endoscopia gástrica mostró concreción de cabellos y moco que ocupaba casi todo el lumen del estómago.

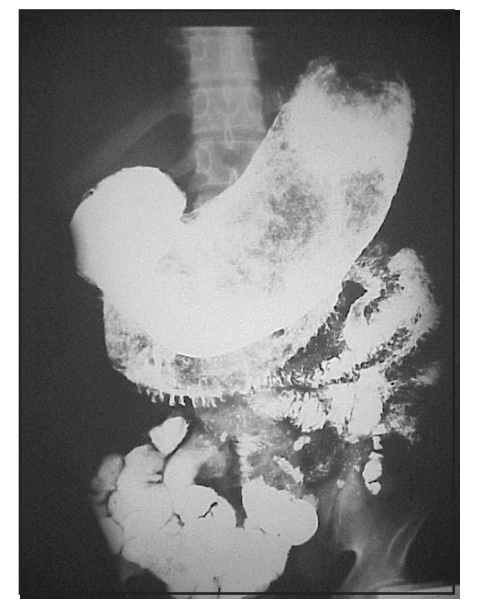

Figura 1.- Serie gastroduodenal mostrando defecto de relleno. 


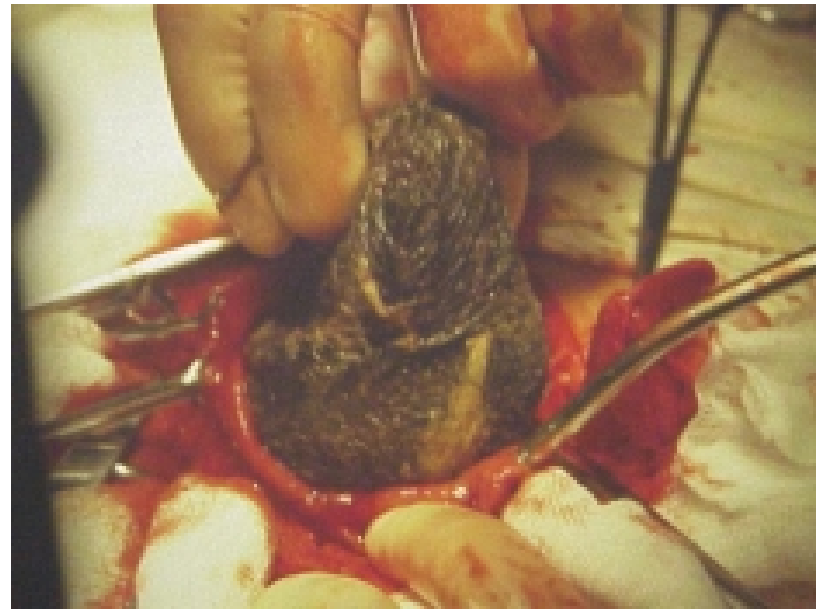

Figura 2.- Gastrotomía: El tricobezoar ocupa toda la cavidad gástrica.

En la evolución, la paciente cursó con cuadro de oclusión intestinal. Fue sometida a una laparotomía con gastrotomía (Figura 2) y extracción de un cuerpo extraño de color negro brillante, fétido, de $30 \times 15 \times 10 \mathrm{~cm}$, que pesó $1800 \mathrm{~g}$ (Figuras 3 y 4 ).

EI postoperatorio fue satisfactorio y sin complicaciones al alta.

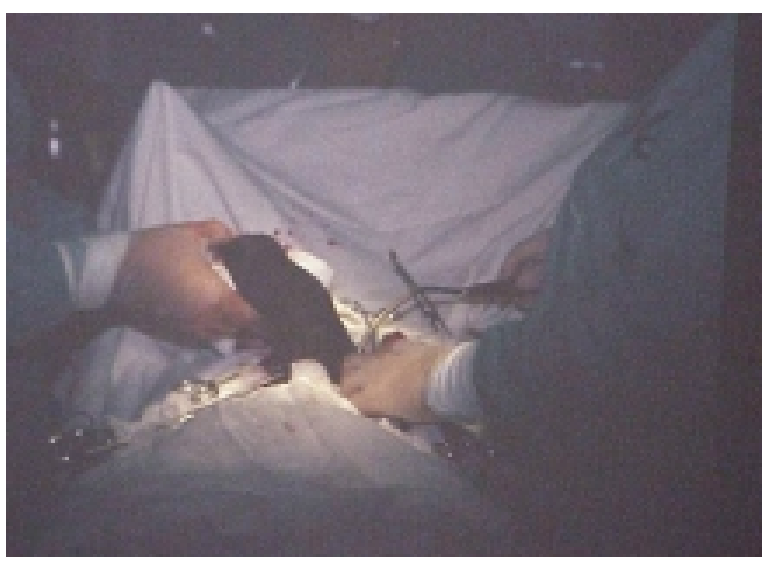

Figura 3.- Gastrotomía: Parto del bezoar.

\section{DISCUSIÓN}

El término bezoar se deriva de las palabras badzher del árabe, del turco panzehir, del persa padzhar y del hebreo beluzzar, que significan antiveneno. Se creía que los bezoares, que eran obtenidos de los animales, tenían poderes curativos $(3,5)$.

En el recuento histórico, se tiene información de la entidad desde el siglo II y III A.C. En el siglo XII A.C., a la piedra bezoar obtenida del estómago de una cabra se le atribuyó propiedades medicinales y fue empleada para tratar diversas enfermedades, como la epilepsia, la disentería, la peste y la lepra, hasta inicios del siglo XVIII. Fue tal su valoración que una de estas piedras forma parte de las joyas de la corona de la R eina I sabel I. En 1779, Baudaman publicó el primer caso, y la primera intervención quirúrgica fue realizada por Schonbern, en $1883(3-5)$.

Los tricobezoares son usualmente encontrados en el estómago, pero cuando atraviesan el píloro y compromete el duodeno, yeyuno, íleo, válvula ileocecal o incluso el colon, toman la denominación de síndrome de Rapunzel, térmi-

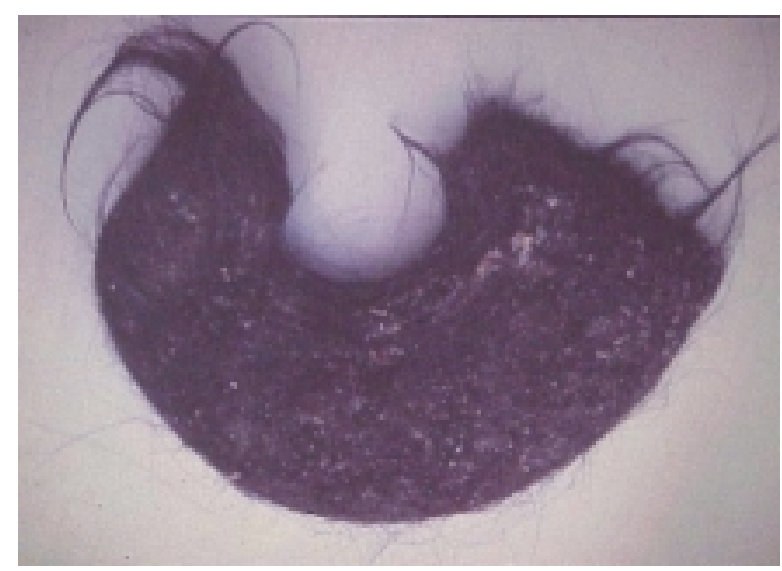

Figura 2.- Especimen de tricobezoar en forma de $\mathbf{J}$. 
no acuñado por $V$ aughan en 1968 (6,7,10,12, 14,16-19). En nuestro caso, el tricobezoar comprometía el estómago y su cola alcanzó hasta la segunda porción del duodeno. Singla, en su reporte del año 1999, habla de SR cuando el tricobezoar se extiende a la válvula ileocecal $\left({ }^{7}\right)$.

Los mecanismos patogénicos no han sido aclarados del todo, pero se estima que algunos factores podrían potenciar la incapacidad del estómago para propulsar y desembarazarse de estos cuerpos extraños antes que se produzca la concreción de los cabellos. Algunos factores predisponentes serían: dismotilidad gástrica, disminución del pH gástrico, alteración de la mucosa y secreción ácida, ingestión de sustancias alcalinas y enzimas pancreáticas, contenido graso en la dieta, posgastrectomía, gastroparesia diabética, distrofia miotónica, autovagotomía secundaria a invasión tumoral (1,3,19).

No está bien definido el tiempo que transcurre entre el comienzo de la tricofagia activa hasta la manifestación sintomática, aunque se supone que en algunos pacientes pueden pasar hasta 15 años, especialmente cuando la ingesta de cabellos es gradual y lentamente progresiva. Sin embargo, se han dado casos de pacientes con tricofagia compulsiva que nunca desarrollaron tricobezoares $(1,20)$.

Uno de cada 2000 niños sufre de tricotilomanía, 5 a $18 \%$ de tricofagia, pero pocos presentan tricobezoares. La incidencia de tricobezoar en pacientes con tricotilomanía no es clara. Tres series de 186, 100 y 24 pacientes con tricotilomanía reportaron bezoares en 0,1 y $37,5 \%$, respectivamente $\left({ }^{2,20}\right)$.

$\mathrm{H}$ abitualmente, el paciente niega u oculta información sobre tricofagia. L a tricofagia es una perversión del apetito determinada por alteraciones af ectivas, aislamiento social, angustia y retraso psicomotor $\left({ }^{20-22}\right)$. Precisamente en el caso que comentamos, la paciente negó el dato de tricofagia; en realidad, el diagnóstico fue hecho por endoscopia y el hallazgo de alopecia estuvo ausente en la historia clínica, como suele ocu- rrir en la mayoría de los casos. El componente depresivo subyacente, pobremente controlado, $y$ el conflictivo entorno familiar fueron determinantes en la conducta asumida, para generar el tricobezoar.

La tricotilomanía puede estar asociada a retraso mental grave o a una condición psiquiátrica, como la esquizofrenia $\left({ }^{21,22}\right)$.

L a tricofagia se relaciona con la pica, que es la conducta de ingerir objetos diversos no alimenticios en forma persistente (por lo menos un mes) e inapropiada para el nivel de desarroIlo del individuo (21-23).

A ntes de los 2 años de vida, es relativamente frecuente observar la ingesta de sustancias no nutritivas, lo que no implica la presencia de pica. Tampoco constituye pica si la conducta de ingestión se da exclusivamente en el contexto de otro trastorno mental, como retraso mental, esquizofrenia, síndrome de Kleine-Levin, síndrome de Prader-W illi (23-25).

Comúnmente se reporta los tricobezoares en mujeres jóvenes; en $90 \%$ de los casos son pacientes de sexo femenino; de ellas, $80 \%$ es menor de 30 años. En la serie de Aird se encuentra un pico de incidencia entre los 15 y 20 años de edad, como corresponde al caso en discusión $(2,5)$.

En una revisión de 311 casos, DeBakey y O chsner encuentran dolor abdominal $(70,2 \%)$, náuseas y vómitos $(64,9 \%)$, debilidad y pérdida de peso $(38,1 \%)$, constipación o diarrea $(32 \%)$ y hematemesis $(6,1 \%)$. F recuentemente presentan marcada halitosis. L a presencia de estos síntomas depende de la elasticidad del estómago, el tamaño del bezoar y de la aparición o no de complicaciones (18,26-31).

En la historia clínica, además debe detallarse antecedentes, como hábito de pica, tricofagia, tricotilomanía, alopecia regional. En el examen físico es recomendable revisar las heces en busca de pelos, examinar el cuero cabelludo buscando zonas de alopecia. U na tumoración ab- 
dominal puede ser palpada en $57 \%$ de fitobezoares y en $88 \%$ de tricobezoares. É sta suele ser grande, móvil, de superficie lisa y firme, localizada en el epigastrio; pero, a veces ocupa posiciones más bajas $\left({ }^{1,4}\right)$. En el caso que comentamos, la palpación de la tumoración asociada al síndrome consuntivo hizo presumir a algún observador de una probable neoplasia. Ocasionalmente, la palpación de esta tumoración se acompaña de crepitaciones. L a incidencia de úlcera gástrica asociada es de $24 \%$ con fitobezoares y $10 \%$ con tricobezoares $\left({ }^{4}\right)$.

Otras formas de presentación clínica menos frecuentes son enteropatía perdedora de proteínas, esteatorrea, poliposis gástrica, ictericia por compresión de la vía biliar, pancreatitis, intususcepción, apendicitis, síndrome de la arteria mesentérica superior, úlcera gástrica traumática ( $10 \%$ con tricobezoar y $24 \%$ con fitobezoar), provocada por la abrasión constante de la mucosa por la concreción pilosa $(4,12,32,33)$.

El diagnóstico se realiza a través de ultrasonografía, radiología con bario y gastroscopía. En la ecografía se puede apreciar aire atrapado alrededor de las fibras de pelo, que origina áreas de ecogenicidad que ocultan la masa, pero perfila la forma del estómago $(2,4,11,12,17,26,31,34)$.

La serie gastroduodenal cuenta con hallazgos radiológicos típicos, definidos por un defecto de relleno intragástrico y, en ocasiones, por la filtración de bario a través de verdaderos desfiladeros gástricos hacia el duodeno (26,34) (F igura 1). La sustancia de contraste que queda embebida en el tricobezoar reproduce una sombra moteada persistente y de alta densidad durante los días posteriores al estudio ${ }^{(26)}$.

L a gastroscopia es la mejor técnica para diagnosticar y clasificar los bezoares. Los tricobezoares son de color negro brillante. La mayoría de fitobezoares varían de color desde el amarillo hasta el verde o marrón. La biopsia endoscópica mostrando cabellos o fibras vegetales es patognomónica ( ${ }^{35}$ ).
El objetivo del tratamiento es su remoción y prevención de la recurrencia que se da en el $20 \%$ de los casos. La mortalidad en el tratamiento quirúrgico del tricobezoar es de $5 \%\left({ }^{2,14}\right)$.

El tratamiento de todos los bezoares, excepto los más pequeños, es quirúrgico, mediante gastrotomía y, en algunos casos, enterotomía por vía abdominal anterior $(11,14,16)$.

La gastrotomía debe ser longitudinal al eje mayor del estómago, lo suficientemente amplia como para provocar el parto del bezoar (Figura 2), observando el estado de la mucosa gástrica para que no queden fragmentos de pelo en los pliegues gástricos, en el intestino delgado y, especialmente, en la válvula ileocecal, donde se acumulan y provocan oclusiones. Se ha descrito tricobezoares de hasta 3250 gramos $\left({ }^{4}\right)$. EI de nuestro caso alcanzó una notable proporción, que ocupaba todo el estómago y parte del duodeno, llegando a pesar $1800 \mathrm{~g}$ (Figuras 3 y 4).

En casos de úlcera gástrica asociada, la remoción del cuerpo extraño es suficiente.

La apertura gástrica se cerrará en dos planos. Las maniobras quirúrgicas y el cierre parietal se deberá realizar pensando que estos pacientes pueden requerir nuevas intervenciones si no mejoran su conducta psicológica $\left({ }^{14,16}\right)$.

El tratamiento en el postoperatorio debe contemplar la desfuncionalización gástrica, dieta gastroprotectora al recuperar el tránsito intestinal, medicación antiácida y psicoterapia $(2,14)$.

No hace mucho se ha reportado resultados alentadores con diferentes métodos endoscópicos, incluyendo fragmentación con láser $\mathrm{Nd}$ :YA G, taladro endoscópico, litotripsia el ectrohidráulica o litotripsia extracorpórea con ondas de choque; pero, la eliminación endoscópica es tediosa, requiriendo de varias sesiones $\left({ }^{36,37}\right)$.

Para bezoares sólidos, el uso del bisturí-aguja modificado (bezotomo en tricobezoar) y un litotrisor modificado (bezotriptor, en fitobezoar) han probado ser útiles $\left({ }^{4}\right)$. 
Otros métodos usados en el tratamiento de fitobezoar lo constituyen las enzimas proteolíticas, como la papaína (37\% de éxito), $50 \%$ para los mucolíticos con acetil cisteína, $83 \%$ para los celulíticos (celulosa) y $85 \%$ para la endoscopia $(1,2,4)$.

El diagnóstico diferencial de la tumoración en epigastrio es con el pseudoquiste pancreático, el neuroblastoma, el riñón en herradura, los tumores gástricos, la hepatomegalia y la esplenomegalia $(2,32)$.

En conclusión, los tricobezoares son acúmulos de cabellos en el estómago en relación a tricofagia. El típico paciente es una adolescente que presenta alopecia y tumoración abdominal móvil en el epigastrio, que puede causar obstrucción intermitente. Un grupo de pacientes es portador de patología psiquiátrica asociada. Cuando el cabo o cola del cabello se extiende a partir de la tumoración principal de localización gástrica hacia el intestino, toma la denominación de síndrome de R apunzel. La presentación clínica incluye dolor abdominal, hiporexia, pérdida de peso, vómitos, pancreatitis, ictericia, anemia e hipoalbuminemia. Este cuadro puede sugerir un proceso neoplásico, pero el diagnóstico de tricobezoar lo da la endoscopia. Las complicaciones del bezoar incluyen úlcera, perforación intestinal, obstrucción e intususcepción. El tratamiento es quirúrgico, cuando la remoción endoscópica fracasa.

\section{BIBLIOGRAFÍA}

1. Florentino J, O neto A, Salgueiro F, Cassella R. Tricobezoares: U na rara entidad con implicancias quirúrgicas [publicación en línea] 2003 [fecha de acceso 24 de abr 2003]. Disponible en: http://www. paideianet.com.ar/ trico.htm

2. Gutierrez J 0. Tricobezoar gástrico.Rev Colomb Cirugía 2000; 15(1): 30-2.

3. Alvarado R, Uribe E, G allego J. Tricobezoar. Presentación de dos casos. A cta Pediatr M ex 2001; 22(4): 264-7.

4. Kishan A, Kadli N, Ponnapa Bg, Korath M, J agadeesan K. Bezoars. [publicación en línea] 2001 [fecha de acceso
24 de abr 2003]. Disponible en: http://www. bhj. org/journal/ 2001_4304_oct/org_507.htm

5. Velasco B, Paredes R. Tricobezoar: Un problema psicológico. An Esp Pediatr 2001; 55: 383-4.

6. Curioso WH, Rivera J, Curioso WI. Síndrome de Rapunzel: Reporte de un caso y revisión de la literatura. Rev Gastroenterol Perú 2002; 22: 168-72.

7. Singla S, Rattan K, Kaushik N, Pandit SK. Rapunzel syndrome-a case report. A m J Gastroenterol 1999; 94(7): 1970-1.

8. Uckun A, Sipahi T, Igde M, Uner C, Cakmak O. Is it posible to diagnose Rapunzel syndrome pre-operatively? E ur J Pediatr 2001; 160(11): 682-3.

9. Hirugade ST, Talpallikar M, Deshpande A, G avali J S, Borwankar SS. R apunzel syndrome with a long tail. Indian J Pediatr 2001; 68(9): 895-6.

10. Sarin Y K. Rapunzel síndrome. Indian Pediatr 1998; 35(7): 682-3.

11. Dalshaug G, Wairser S, Hollaar G. The Rapunzel syndrome (trichobezoar) causing atypical intussusception in a child: A case report. J Pediatr Surg 1999; 34(3): 479-80.

12. Kaspar A, Deg K H, Schmidt K, Meister R. Rapunzel syndrome, an rare form of intestinal trichobezoars. Klin Padiatr 1999; 211(5): 420-2.

13. Seker B, Dilek 0, Karaayvaz M. Trichobezoars as a cause of gastrointestinal obstructions:T he Rapunzel syndrome. A cta Gastroenterol Belga 1996; 59(2): 166-7.

14. Uroz J, G arcia X, M elian S. Rapunzel síndrome: A report of new case. Cir Pediatr 1996; 9: 40-1.

15. Pul N, Pul M.The Rapunzel síndrome (trichobezoar) causing gastric perforation in a child: A case report. Eur J Pediatr 1996; 155(1): 18-9.

16. Balik E, UIman I, Taneli C, Demircan M. The Rapunzel síndrome: A case report and review of the literature. Eur J Pediatr Surg 1993; 3(3): 171-3.

17. Duncan N, Aitken R, Venugo Pal S, West W, C arpenter $\mathbf{R}$. The Rapunzel síndrome. Report of a case and review of the literature. W est Indian M ed J 1994; 43(2): 63-5.

18. Wolfson PJ, Fabius RJ, Leibowitz AN. The Rapunzel syndrome: An unusual trichobezoar. A m J Gastroenterol 1987;82(4):365-367

19. Sánchez W, Rodríguez $\mathbf{D}$, L una-Pérez $\mathbf{P}$. Síndrome de Rapunzel (tricobezoar). Rev Gastroenterol M ex 1997; 62(4): 284-6.

20. Chaudhury S, John T, G hosh S. Recurrent trichobezoar in a case of trichotillomania. Ind J Psichiatry 2001; 43(4): 340-1.

21. Bouwer C, Stein D. T richobezoars in trichotillomania: Case report and literature overview. Psychosomatic M ed 1998; 60: 658-60.

22. Sharma NL, Sharma RC, Mahajan VK, Chauhan D, Sharma AK. Trichotillomania and trichophagia leading to trichobezoar. J Derm 2000; 27: 24-6.

23. Ruiz W. Pica, un enigma aun por resolver: Rev Per Soc $\mathrm{M}$ ed Intern. 2001; 14(3): 127-38. 
24. Couper R. Clinical picture: Rapunzel syndrome. The $L$ ancet 2001; 358(9290): 1304.

25. $R$ ajaonarison $\mathbf{P}, \mathbf{R}$ alamboson $\mathbf{S}, \mathbf{R}$ amanampamonjy $\mathbf{R}$, $R$ akotoarisoa $R$, $R$ amanantoanina CE, Andrianasolo $D$, Andriantsimahavandy A. Le trichobézoard, une entité clinique peu courante. Arch Inst Pasteur de M adagascar 2001; 67(1-2): 65-7.

26. W eiss M, Danoff D, Wood B. Radiological case of the month: Jejunojejunal intussuception caused by a trichobezoar with a tail. Arch Pediatr Adolescent M ed 1998; 152(4): 1-5.

27. O'Sullivan M, Mcgreal G, Walsh J, Redmond HP. Trichobezoar. J R Soc M ed 2001; 94: 68-70.

28. Varma A, Sudhindra BK. Trichobezoar with small bowel obstruction. Indian J Pediatr 1998; 65: 761-3.

29. Qureshi N, Morris K, McDevitt B. Trichobezoar - a condition to think of in case of mobile abdominal mass. Irish Med J. 1992; 85(2): 73-4.

30. Pozo J, Gómez T, Rincón N, Berrios C. Tricobezoar: diagnóstico inusual. Reporte de tres casos. GEN 1995; 49(2): 157-60.
31. B arzilai M, Peled N, Soudack M, Siplovich L. Trichobezoars. Harefuah 1998; 135(3-4): 97-101.

32. A zuara-Fernáandez H M, Azuara-Gutierrez $H$, Hernández-Márquez $\mathrm{N}$, R evilla-C epeda $\mathrm{E}$, A portela $\mathrm{A}$, Andrade-Sepulveda VR. Intestinal Trichobezoar: Differential diagnosis in children with an abdominal mass. Bol M ed Hosp Infant M ex 1989; 46(11): 732-5.

33. A ytac B, C akar S. Bezoar: A n uncommon cause of intestinal obstruction. Acta Gastroenterol Belg 2001; 64(3): 295-7.

34. Narinder K, Sharma Y, Negi A, Jaswal A. Images Gastric trichobezoar. Ind J Radiol Imag 1999; 9(3): 137-9.

35. Alsafwah S, Alzein M. Small bowel obstruction due to trichobezoar: Rol of upper endoscopy in diagnosis. Gastrointest Endosc 2000; 52: 784-6.

36. L ubke HJ, Winklenmann RS, Berges W. Gastric phytobezoar endoscopic removal using the gallstone lithotriper. Gastroenterol 1988; 26: 393-6.

37. B enes J, C hmel J, J odl J. T reatment of gastric bezoar by extracorporeal shock wavelithotripsy. Endoscopy 1991; 23: 346-8. 